\title{
Neutrino Temporal Oscillation
}

\author{
Russell Bagdoo \\ Saint-Bruno-de-Montarville, Quebec, Canada \\ Email:rbagdoo@gmail.com,rbagdoo@yahoo.ca
}

How to cite this paper: Bagdoo, R. (2021) Neutrino Temporal Oscillation. Journal of Modern Physics, 12, 513-535.

https://doi.org/10.4236/jmp.2021.124034

Received: January 5, 2021

Accepted: March 16, 2021

Published: March 19, 2021

Copyright (c) 2021 by author(s) and Scientific Research Publishing Inc. This work is licensed under the Creative Commons Attribution International License (CC BY 4.0).

http://creativecommons.org/licenses/by/4.0/

\section{Abstract}

We conjecture the existence of massless neutrinos that are in the line of Standard Model (unable to account for the neutrino mass) but have characteristics that are not accounted for the Standard Model: they use a shorter radial path than the photon and possess bosonic flavors, considered like bosons instead of fermions. We call this theory "neutrino temporal oscillation". Faced with some experimental comparisons solar neutrinos, neutrinos from
\end{abstract} SN 1987A, cosmological neutrinos, the theory gives better results, explanations and sense than the complicated theory of neutrino oscillations (transformism). The deficit of detection of solar neutrinos would have been blindly attributed to the "neutrino oscillation" by physicists who quickly concluded that the neutrino and the photon follow the same transverse path. The "OPERA" experiment which measured the speed of neutrinos in 2011 resulted, after a "superluminal" saga, in neutrino speeds consistent with the speed of light, in data that the three existing types of neutrinos cannot explain, with the final outcome of a fourth "sterile" neutrino with non-standard interaction. OPERA findings aren't just in conflict with existing theory, but other measurements as well. For example, a study from the Kamiokande II experiment in Japan of the supernova SN1987A found that light and neutrinos that departed this exploded star arrived at Earth within hours of each other. Even though measurements of the neutrinos emitted by this supernova strongly suggest that their speeds differ from light by less than one part in a billion, the fact remains that two types of data were collected, and that only one was retained to be consistent with the existing theory. Thus, the OPERA observation is in conflicts with the result of SN1987A, which itself is highly doubtful. And what about the neutrinos and antineutrinos born during the big bang, except that they were never detected and there is nothing to indicate that their speed could be other than that of light. Neutrino physics seems sick, belief is transformed into evidence. The theory of "Neutrino temporal oscillation" shows hint that massless neutrinos can take a shortcut through the three spatial dimensions of the space-time that we know. It represents within the Standard Model an open window on a "new physics" that has a connection 
with physical reality.

\section{Keywords}

Apparent Superluminal Neutrinos, General Relativity, Neutrino Oscillation, Neutrino “Temporal” Oscillation, “Bosonic” Flavors, Radial Path (Longitudinal), Transverse Path, Longitudinal Waves $t_{o} c$ of the Neutrino, Transverse Waves tc of the Photon, False Flux of Neutrinos, True Flux of Neutrinos

\section{Introduction}

\subsection{History of the Neutrino}

In 1930, Wolfgang Pauli rescues conservation of energy by hypothesizing an unseen particle that takes away energy missing from some radioactive decays. Enrico Fermi in 1933 formulates the theory of beta-decay incorporating Pauli's particle, called the neutrino (little neutral one). Frederick Reines and Clyde Cowen first detect the neutrino in 1956 and at Brookhaven in 1962 the first accelerator beam of neutrinos proves the distinction between electron-neutrinos and muon-neutrinos. In 1969, Raymond Davis, Jr., first measures neutrinos from the Sun, using 600 tons of cleaning fluid in a mine in Homestake, S.D. The tau lepton and $b$ quark are discovered in 1975-1977, revealing a third generation of quarks and leptons. $W$ and $Z$ bosons are discovered at CERN in 1983: they are carriers of the weak force, which mediates neutrino reactions. The $Z$ decay rate was measured at SLAC and CERN in 1989, showing there are only three active neutrino generations. In 1987, the IMB and Kamiokande proton decay experiments detect 19 neutrinos from Supernova 1987A in the Large Magellanic Cloud [1].

\subsection{The Theory of Neutrino Oscillations}

The theory of neutrino oscillations arises in the late 1990s. Neutrinos were found to have mass and a speed under the light speed after having thought the opposite for decades. Since then, neutrinos metamorphose: they shift among three known neutrino types. As they propagate at nearly the speed of light through space, the celestial bodies, or our body, they often change identities, oscillating between three varieties, or "flavors", the electron, the muon and the tau. Quantum mechanics permits neutrinos to oscillate between flavors only if they have mass and if each flavor has a different mass. Super-K in 1998 assembles evidence of neutrino oscillation using atmospheric neutrinos [2].

\subsection{Cosmic Mismatch Hints at the Existence of an Enormously Heavy Neutrino or a Lightly Sterile Neutrino}

All neutrinos are classified as leptons, meaning that they do not feel the strong force and, lacking electrical charge, they do not feel electromagnetic forces, ei- 
ther. That leaves the weak interaction and the force of gravity for the three known flavors. Neutrinos must be left-handed to feel the weak force, responsible for radioactive decay. Theorists know that they have mass (a rest mass referring to the mass that matter is made out) but not how much, that they come in at least three flavors but there may be more. They hint that a fourth type of hitherto unseen neutrino exists. Even if particles physicists would prefer a new type of neutrino enormously heavy, theorists perceive them with a little bit of mass, enough to have the ability to swap flavors [3]. Very sensitive experiments have revealed that neutrinos do have a very small non-zero rest mass: in 2019, KATRIN (Karlsruhe Tritium Neutrino experiment) scientists estimated that the range for the rest mass of the neutrino is no larger than about $1 \mathrm{eV}$. Therefore, they travel at a speed very close to $c$ but slightly lower.

\subsection{Neutrinos with Zero Rest Mass}

In 2007, an experiment on neutrinos created at Fermilab in Illinois and beamed through the Earth to the Soudan Mine in Minnesota showed that the neutrino speeds were consistent with the speed of light. Measurements of neutrinos emitted from a supernova in the Large Magellanic Cloud in 1987, moreover, suggested that their speeds differed from light by less than one part in a billion. This suggests the existence of neutrinos without rest mass, as originally planned. Once thought to be massless and to travel at the speed of light, the neutrinos can sail through walls and planets like wind through a screen door. By Einstein's equation $E=m c^{2}$, a particle's total energy or mass-energy includes the particle's rest mass and momentum. When a nucleus goes through the process of beta decay, the electrons that are emitted have a range of kinetic energies. This variation confirms that there is an extra particle in the mix. If neutrinos have a nonzero rest mass, then the very high end of the electron energy spectrum will be slightly distorted, and the highest electron energy will be less than the maximum possible energy by a very small amount-the tiny mass of the neutrino. So far, investigators have managed to observe only a non-significant distortion at the end of the energy spectrum. Therefore we still can consider that a moving neutrino's mass-energy comes mainly from its momentum [4]. Neutrinos are massless in the Glashow-Salam-Weinberg Standard Model [5] [6] [7].

During the years 2009 to 2011, neutrino beams were fired repeatedly from CERN towards a detector in Italy's Gran Sasso tunnel, some $4^{\circ}$ south and $7^{\circ}$ east of CERN, at a distance of $730 \mathrm{~km}$, in the shape of short bunches of particles. Their time of flight $(2.5 \mathrm{msec})$ was measured at high accuracy (ns) with caesium clocks. In 2011, the CNRS team found a deficit of $\sim 57.8 \mathrm{~ns}$ compared with propagation at the speed of light, and announced a superluminal speed. In 2012, the Italian OPERA scientists reported that the neutrinos "respect the cosmic speed limit" and that there was an error in the speed measurement due to an incorrectly screwed cable of the experiment's fiber-optic timing system [8]. It turns out the master clock in charge of keeping time for the experiment was also 
improperly calibrated. Ironically enough, this miscalibration would make the neutrinos appear to travel slower, but this error wasn't large enough to cancel out the faulty cable. Accounting for these two sources of error eliminated the faster-than-light results. The difference between the measured and expected arrival time of neutrinos (compared to the speed of light) was approximately $6.5 \pm$ $15 \mathrm{~ns}$. Thus, the speed of neutrinos is consistent with the speed of light within the margin of error. It was the end of the apparently faster-than-light neutrino anomaly for most scientists.

For some, it is always an open question. We agree that the speed of neutrinos is consistent with the speed of light. But the difference between the measured and expected arrival time of neutrinos, although it was greatly reduced compared to the initial results (from $\sim 57.8 \mathrm{~ns}$ to $\sim 6.5 \pm 7.4 \mathrm{~ns}$ ), is still longer than the expected time of the neutrinos. This is an indication that even if there is no faster-than-light neutrino speed, the neutrinos followed a shorter path and makes our theory plausible.

Even though OPERA's counter-expertise is convincing, its findings do not fall within the margin that would make the faster-than-light appearance indefensible. Often theorists take the results of some retests for granted because they reinforce their definitive conceptions of science. We have set out this point of view in the article Recycled Relativity [9]. In this regard, we present in Section 2 an ad hoc formula for the CERN apparently superluminal neutrino while respecting the principle of the speed limit of light. In Section 3 we submit the theory of neutrino temporal oscillation which incorporates the longitudinal wave. In section 4 we confront this theory with the experimental observations relating to the solar neutrino, the supernova $1987 \mathrm{~A}$ and the cosmological neutrino. We suggest the new "bosonic" flavors. Discussion in Section 5: Analysis of the proposed theory where neutrinos at the speed of light follow a shortcut in space-time and of the current theory where massive neutrinos metamorphose by moving with a speed below the speed of light. Finally, we conclude in Section 6 .

\section{Ad Hoc Formula for the CERN Apparent Superluminal Neutrinos}

Are we going to believe a measure of complacency? Recall that the physics is not completed, as well as those experiences, and we want to explore the hypothesis that neutrinos detected by Opera have apparently traveled faster than light, while respecting the inviolability of the speed of light which is the pivot of relativity. We suggest the following ad hoc formula which gives the apparent drift of the supraluminal neutrino without violating the sacred principle of speed light invariance

$$
v_{o}=c /\left(1-v^{2} / c^{2}\right)^{1 / 2}=c /\left[1-G M_{E} /\left(R \sin x c^{2}\right)\right]^{1 / 2} .
$$

$\left(G M_{E} / c^{2}\right.$ is the Schwarzschild radius of Earth, or the interval the space $d s^{2} ; v_{o}$ is the apparent superluminal speed of the neutrino; $c$ is the static speed of propa- 
gation; $v$ is the velocity of the source; $R$ is the distance done by the photon between the emitter and the receiver; $R \sin x$ is the distance traveled by the neutrino between the transmitter and the receiver.)

Since we know the distance $(730 \mathrm{~km})$, the journey time (2.4 milliseconds) and the anticipation of neutrinos of 60 nanoseconds on this distance compared with photons, one can express $60 \mathrm{nsec}$ in terms of distance on the distance of $730 \mathrm{~km}$, or $18.25 \mathrm{~m}(750 \mathrm{~km} \times 60 \mathrm{nsec} / 2.4 \mathrm{msec})$.

We assume that the apparent superluminal velocity of the neutrino at the end of the trip of 2.4 milliseconds is at least $299,792,476.3 \mathrm{~m} / \mathrm{s}$

$\left(v_{o}=c+18.25 \mathrm{~m}=299792476.3 \mathrm{~m} / \mathrm{s}\right)$.

$299792476.3 \mathrm{~m} / \mathrm{s}=c /\left(1-v^{2} / c^{2}\right)^{1 / 2} ; v^{2}$ is equivalent to $105 \mathrm{~km} / \mathrm{s}$ squared. In general relativity, $v^{2}$ acts as gravitational potential.

$$
299792476.3 \mathrm{~m} / \mathrm{s}=c /\left(1-\Phi / c^{2}\right)^{1 / 2}=c /\left[1-G M_{t} /\left(R c^{2}\right)\right]^{1 / 2}
$$

Although $G M_{E} / c^{2}$ is the Schwarzschild radius, or the interval of space $d s^{2}, R$ is not the radius of the Earth but represents a journey of $730 \mathrm{~km}$ that a photon would do if he left from the Earth's center. However, a neutrino would not follow the transversal path of the photon but a radial path that would be $730 \sin x$ $\mathrm{km}$.

$$
\begin{aligned}
299792476.3 \mathrm{~m} / \mathrm{s} & =c /\left(1-G M_{t} / 730000 \sin x c^{2}\right)^{1 / 2} \\
& =c /\left[1-G 5.98 \times 10^{24} \mathrm{~kg} /\left(730000 \sin 2.85^{\circ} c^{2}\right)\right]^{1 / 2}
\end{aligned}
$$

$x=2.85^{\circ}$ indicates a radial path. $730,000 \sin 2.85^{\circ}$ reduces to $36.3 \mathrm{~km}$ the path radially traveled by the neutrino at speed apparently superior to light.

We can imagine with difficulty that a neutrino crosses radially $36.3 \mathrm{~km}$ through the superimposed curvatures of the Earth to arrive slightly before a photon having traveled $730 \mathrm{~km}$. This formula is not a guarantee of validity, but an interesting mathematical option. It has the merit of leaving the speed apparently faster than the speed of light and illustrates the trend of the neutrino to follow a shortened radial path rather than a transverse path. Without significant comparison with what follows, except for the shortened course, it preludes the "neutrino temporal oscillation" which stands out with a non-exceeded speed of light.

\section{Theory of Neutrino Temporal Oscillation}

\subsection{Uncertainty of Neutrino Oscillations}

Scientists of neutrino do not know its mass, its energy, the distance it travels, and if they know how much between two points they are unable to tell what path he traveled. Moreover, they ignore its speed. What do they know? Statistical averages. And a nice theory, the neutrino oscillations, which says that the neutrino has a mass and this ability to metamorphose. Like all elementary matter particles, they come in three versions, called flavors. The electron (e) has two heavier replicas, the muon $(\mu)$ and the tau $(\tau)$, and each has a neutrino partner: the 
electron-neutrino $\left(V_{e}\right)$, the muon-neutrino $\left(V_{\mu}\right)$ and the tau-neutrino $\left(V_{\tau}\right)$. But whereas the electron, the muon and the tau have specific masses, the three neutrino flavors do not. If you measure the mass of a neutrino with a given flavor, you get one of three answers at random, with a certain probability for each. Conversely, if you measure the flavor of a neutrino with a given mass, you get one of three answers. A neutrino can have either a specific flavor or specific mass but not both at the same time. Neutrinos thus violate a basic intuition we have about objects [10]. With the glorious uncertainty of this dominant theory, an alternative or complementary theory would not be superfluous.

\subsection{Neutrino Temporal Oscillation}

We term "temporal oscillation" an economy of time generated by a wave intrinsically less broad than the standard wave. It is also a quantum mechanical phenomenon whereby a neutrino created with a specific bosonic flavor (neutrino-photon, neutrino-graviton) can later be measured to have a different flavor. Flavors will be addressed in Section 5 . We begin by the first part of the definition.

\subsection{What Is the Shortest Line? Transversal Way for the Photons and Shorter Longitudinal Way for the Neutrinos}

Right now the path taken by a photon actually defines what a straight line is. But is it the shortest distance between two points? Concerning an undulation, we think it is the longest path. Grossly, we consider two kinds of paths for a particle at the speed of light: transversal way and longitudinal way (or radial). Science picks up electromagnetic waves and so measures the universe. They follow a transversal path. There is a transverse wave when the oscillatory motion of any part of the system is at right angles to the direction in which the wave is traveling. There is a longitudinal wave when the oscillatory motion of a part of the system is in the same direction that the wave is traveling.

The sinusoid ACBDE of the following drawing shows two semi-circumferences $\mathrm{ACB}$ and BDE. If we put the two half-circumferences of the sinusoid directly on top of each other, they form a concentric circle. The diameter $d\left(\mathrm{AOB}=\mathrm{BO}^{\prime} \mathrm{E}\right)$ divides the circumference and the circle into two equal parts. The radius $\mathrm{OC}$, $\mathrm{OB}, \mathrm{O}^{\prime} \mathrm{B}, \mathrm{O}^{\prime} \mathrm{D}$ are equal.

The photon follows a transverse wave at the speed of light. Its measurement between $\mathrm{A}$ and $\mathrm{E}$ is that of the sinusoid $\mathrm{ACBDE}$, or the circumference of the concentric circle having $\mathrm{O}$ (or $\mathrm{O}$ ) at the center. We postulate the existence of a sort of neutrinos with a mass equal to the mass of light, the lightest known mass. The measurement of the neutrino at the speed of light between $\mathrm{A}$ and $\mathrm{T}$ (we use $\mathrm{T}$ of the word Time in Figure 1) is the radial line (or longitudinal) AOBO'ET. When the photon has traveled the metric of the sinusoid ACBDE equivalent to the circumference of a circle with $\mathrm{O}$ (or $\mathrm{O}^{\prime}$ ) at the center, the neutrino has traveled radially $\pi d$, as if we undid the circumference for stretch it in a straight line (Figure 2). We associate the photon to the circumference and the neutrino to the diameter. 


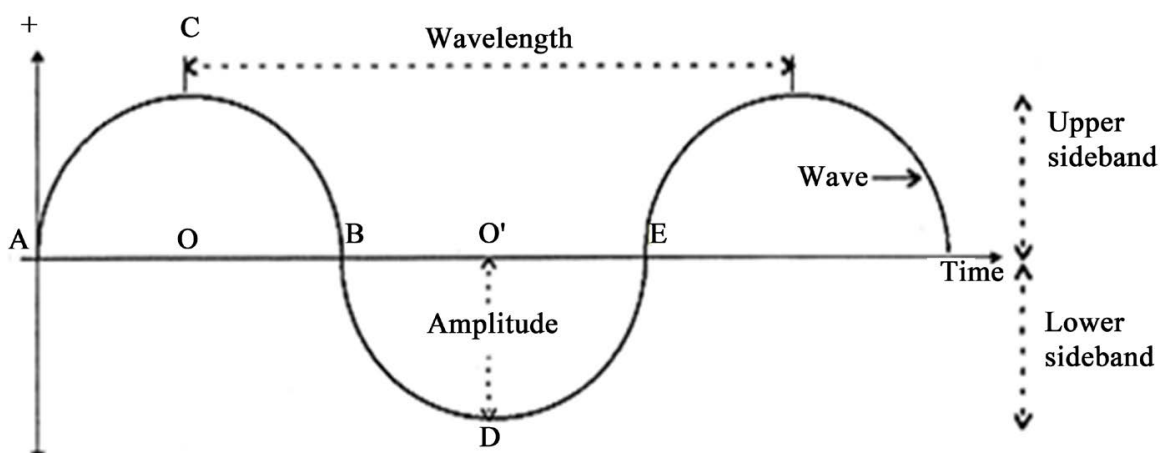

Figure 1. Sinusoid. (While the photon follows the transverse wave $t_{0} c$ on the sine wave ACBDE (equivalent to a circle) at the speed of light, the neutrino follows the longitudinal wave $t c$ along the radial sinusoid AOBO'ET(equivalent to $\sim \pi$ diameter) at the speed of light. The ordinary transverse second of the photon is $\sim \pi$ times longer than the longitudinal second of the neutrino: 1 second $t=\sim \pi$ second $t_{0}$.)

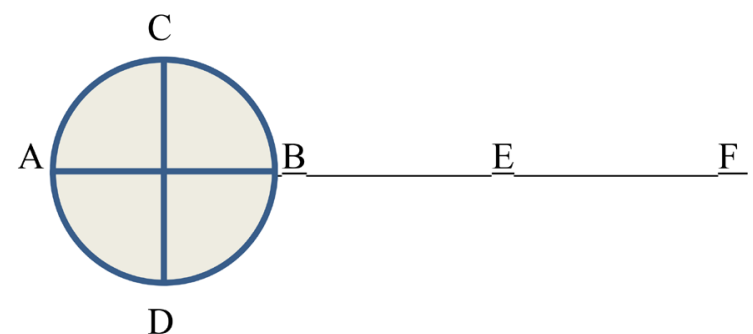

Figure 2. "Radial" sinusoid. (The longitudinal wave $t_{o} c$ of the neutrino follows the flattened sinusoid ABEF while the transverse wave $t c$ of the photon follows the sinusoid ACBDE of Figure 1, equivalent to the ACBD circumference in Figure 2. The neutrino is associated with diameter. The photon is associated with the circumference.)

In other words, we pretend that the massless neutrino follows the longitudinal way (or radial) while the photon follows the transversal way. The term $t_{o} \mathrm{c}$ represents a longitudinal wave and $1 \sec \left(t_{o}\right)$ is the second of the neutrino associated with the longitudinal way. The term $t c$ means a transverse wave and $1 \mathrm{sec}$ $(t)$ is the second of the particle associated with transversal way. In circular time (or Newtonian time), which is the one we use, 1 second corresponds to $\pi$ linear seconds: $1 \mathrm{sec}(t)=\pi \sec \left(t_{o}\right)$. The ordinary transverse second $t$ is 3.1416 times longer than the longitudinal second $t_{0}$. Both particles go to the speed of light, so

$$
t_{o} c / t_{o}=\pi t_{o} c / \pi t_{o}=t c / t=c .
$$

This means that if a particle has to travel the distance $\mathrm{AB}=\lambda=t_{0} c$, it will take $t_{o}$ time. Then the wave and the particle are propagating at the speed of light, and the direction in which the wave is traveling and the line of the oscillatory motion of the particle are making one line. But if the oscillatory motion of the particle is at right angles to the direction in which the wave is traveling, the time required for the particle to travel from $\mathrm{A}$ to $\mathrm{B}$ is $\pi t_{o} c$, because the particle is covering $\pi t_{o} c$ distance, running in circle around the line $A B$. Note that we should say about $\pi$, or about 3 , because we must envision the encirclement of a spiral structure instead of a closed two dimensions circle. 


\subsection{Discussion: Range of Longitudinal Waves and the Rule of the Displacement of the Nodes Due to the Inverse Sine}

We suppose that in most of the cases, the neutrino follows a different path from the photon to browse the same distance, even if it keeps the speed of light. It means that the sinusoid traveled by the neutrino flattens, becomes "radial", what drives knots further away on the straight line AF (Figure 2). The sinusoid being completely spread over the radial line, we can say that when a photon travels a radius with regard to a circumference, the neutrino browses linearly $\pi$ time this distance, what amounts to a half-circumference; when the photon travels a diameter, the neutrino travels radially $\pi$ diameters, i.e. equivalent to a circumference.

Most neutrino physicists believe that the neutrino follows the same path the photon and that the transverse path of the photon is the straight line. That is not supported by the fact that according to our theory of temporal oscillations, the longitudinal path of the neutrino turns out to be the straight line. It follows that an observer B, who anticipates to receive from A in one ordinary second (transverse), a full neutrino flux will be surprised to receive just about thirty-three percent of the expected flux, the two other thirds having already reached the $\mathrm{F}$ point. However, it is assumed that there is a range of wavelength between the transverse wave and the radial wave. The current theory of neutrino oscillation (transformism) follows the transverse way perpendicular to the radial direction of the wave. In this case, the neutrino, lively at speed $c$, follows the same sinusoid that the photon between nodes $\mathrm{A}$ and $\mathrm{E}$ on Figure 1 and travels the same metric at the same time. The second of the photon is then equivalent to the second of the neutrino and can be imagined by using a kind of rule of displacement of nodes due to the trigonometric function cosecant defined as the inverse sine. This rule of an angular cosecant is a simple supposition. So, for the time factor:

$$
1 \sec t_{o} \text { for the neutrino }(v) / \operatorname{sine} 90^{\circ}=1 \sec t \text { for the photon }(\gamma) \text {. }
$$

The intent is to show that the photon travels from $\mathrm{A}$ to $\mathrm{B}$ via $\mathrm{C}$ in one transversal sec and that the neutrino flies in the same way from A to B in one transversal sec. Sine $90^{\circ}$ indicates that they follow the same transverse path, or spiral. Considering the distance factor in this case, the photon and the neutrino travel the same distance, we can write

$$
1 \mathrm{~m} t(\gamma) / \operatorname{sine} 90^{\circ}=1 \mathrm{~m} t_{o}(v) .
$$

If we suppose in terms of time a displacement of the nodes due to the inverse sine $85^{\circ}$, we obtain

$$
1 \sec t_{o}(v) / \operatorname{sine} 85^{\circ}=1.0038198 \sec t(\gamma) \text {. }
$$

Thus, we can say that to go from to $\mathrm{A}$ to $\mathrm{F}$ (due to sine $85^{\circ}$ ), the neutrino uses 1 longitudinal sec whereas the photon uses 1.0038198 transversal sec. In terms of length, we can put

$$
1 \mathrm{~m} t(\gamma) / \text { sine } 85^{\circ}=1.0038198 \mathrm{~m} t_{o}(v) .
$$

Consider the 730-kilometre trip from CERN in Switzerland to the Gran Sasso 
underground laboratory in Italy. Suppose that photons and neutrinos start at the same time and make the journey in a straight line. The agreed line is that of the transverse photon. While the photon complete $730 \mathrm{~km}$, the longitudinal neutrino passing through the Earth at light speed would have traveled $2294 \mathrm{~km}(730 \times$ $\pi)$. If we apply in terms of distance the displacement of the nodes due to the inverse sine,

$$
1 \mathrm{~m} t(\gamma) / \operatorname{sine} 18^{\circ} .5607=3.14159 \mathrm{~m} t_{o}(v) .
$$

While the photon travels a circle that seems to merge with a diameter in the radial direction of propagation, the neutrino moves around $3.14159 \mathrm{~m}$; the length of the straight neutrinos is

$$
730 \mathrm{~km} t / \text { sine } 18^{\circ} .56075=2294 \mathrm{~km} t_{o} .
$$

This means that the $730 \mathrm{~km}$ serpentine path of the electromagnetic particle is radially stretched over a distance of $2294 \mathrm{~km}$. In terms of time,

$$
1 \text { sec } t_{o}(v) / \operatorname{sine} 18^{\circ} .56075=3.14159 \sec t(\gamma) \text {, }
$$

which signifies that the photon goes from A to E along a transverse path (sinusoid ACBDE on Figure 1) in 3.1415917 radial sec while the neutrino travels from $A$ to $B$ along a longitudinal path in one radial sec. We can also say that the photon travels from $A$ to $B$ along a transverse path (equal to the straight line ABEF on Figure 2) in $1 \sec t$ while the neutrino goes radially from A to B in $1 \sec t / \pi$.

\subsection{About the Longitudinal Wave}

By scanning the history of longitudinal and transverse waves we notice a kind of cycle, the periods of longitudinal wave which alternate with the periods of transverse wave. The theory of Huygens, contemporary of Newton, was based on a profound analogy between light and sound waves. One hundred fifty years later, Fresnel was led to assume that light does not consist of longitudinal vibrations, such as those of sound in air, as Huygens thought, but transversal, and that alone a special medium having the properties of a hard body could convey them in universal space. Poisson discovered that the waves in an elastic solid are of both kinds: transverse and longitudinal. To rule out the contradictions which, in a series of cases arose from both theories, Maxwell thought that light does not consist of Huygen's longitudinal waves neither of Fresnel's transverse waves of ether, but in waves of an autonomous electromagnetic field. H.A. Lorentz showed that the electromagnetic theory of Maxwell, explained by the mechanical theory of ether, required the introduction, in addition to the light waves, of longitudinal waves of ether [11] [12] [13].

While it is recognized that the longitudinal waves propagate in air, liquid and solid, modern mainstream technology has been optimized to deal solely with transverse waves and is therefore largely incapable of measuring, let alone detecting, longitudinal waves. We still found some books on physical electronic introducing longitudinal theories, such as the longitudinal space-charge wave theory [14]. 
Since Einstein rejected the ether as superfluous, only the transverse waves can propagate in the vacuum. Physicists consider that it is mathematically and geometrically impossible for a longitudinal wave to have both electric and magnetic components simultaneously. For this reason physicists dismiss the possibility of longitudinal $\mathrm{E} / \mathrm{M}$ waves.

A changing voltage field can give rise to concussive waves that are radiated away in the direction of propagation. The fluctuations, with a curl-free vector potential and without magnetic fields, are longitudinal rather than transverse. These longitudinal waves are what Maxwell termed displacement current. They do not violate Maxwell's equations that state there must be an induced magnetic field for every change in the electric field. There is a longitudinal E/M wave when all the magnetic fields cancel and yet there is still a displacement current. Usually current is defined as a flow of charges, but across a capacitor consisting of two conductors separated by an insulator that allows no charge to pass, oscillating energy can still transfer. Aside from a changing voltage field, current flows from a large flat metal plate charged to a steady high voltage can give rise to a steady electric field pointing out and away from the plate in the direction of radiation. The resulting wave that also fluctuates in the direction of propagation is longitudinal.

Maxwell equations allow two possibilities: transverse EM waves and longitudinal $\mathrm{E} / \mathrm{M}$ wave. Longitudinal $\mathrm{E} / \mathrm{M}$ waves are just as real as transverse $\mathrm{EM}$ waves but are more difficult to detect. The assumption that "what cannot be measured does not exist" fails to take into account that the shortcoming might be with technology rather than reality.

\section{Comparisons with Experimental Observations}

\subsection{Solar Neutrino}

In the 1929s and 1930s, scientists proposed [15] [16] [17] that nuclear fusion reactions among light elements occur near the centre of the Sun and provide the energy that the Sun has emitted for four-and-a-half billion years [18]. The simplest of all possible reactions is the nuclear reaction in proton-proton (p-p) collisions, which yields low-energy neutrinos: $\mathrm{H}+\mathrm{H}=\mathrm{D}+e^{+}+v$. The deuterons formed will quickly react further, and the end product of p-p reaction of hydrogen is helium. 98 percent of the Sun energy comes from the nuclear reaction chain p-p [19]. While most of this energy ends up as electromagnetic radiation from the surface, approximately three per cent are believed to be emitted directly from the centre of the Sun in the form of neutrinos [20].

Knowing the energy radiated by the Sun and the part of fusion energy carried away by a neutrino, we easily deduce the amount of neutrinos escaping from the Sun per unit of time. The Sun produces only electron neutrinos. We therefore deduce, aware of the Earth-Sun distance, the theoretical neutrino flux per unit area and per unit time at the level of Earth. The flux of neutrinos at Earth is several tens of billions per square centimetre per second. They cross the entire 
Earth, interact weakly with matter and are difficult to detect. From the characteristics of the detector, the amount of neutrinos that is to be detected per day in this flux is found. All experiments (on different time scales, with many detectors based on different principles) to measure the flow of electronic neutrinos from the Sun showed that the number detected was much lower than predicted. In various experiments, with detectors that became very sensitive, the number deficit was between one half and two thirds.

Few separate experiments to detect neutrinos from the Sun which confirmed a deficit in the flux relative to the predictions of standard theories of nuclear physics, have led to suggestions that neutrinos may have small masses and may oscillate between different types. In 1968, Pontecorvo proposed that if neutrinos had mass, then they could change from one type to another [21]. Essentially, the "missing" solar neutrinos could be electron neutrinos which changed into other types along the way to Earth and therefore were not seen by the detectors in the Homestake Mine in the late 1960s and contemporary neutrino observatories. Thus, the discrepancy between measurements of the numbers of neutrinos flowing through the Earth and theoretical models of the solar interior, lasting from the mid-1960s to about 2002, has since presumably been resolved by new understanding of neutrino physics, requiring a modification of the Standard Model of particle physics - specifically, neutrino oscillation.

That being said, we believe that a significant alternative could explain why the measurements of solar neutrino fluxes all agree with theoretical expectations to within a factor of two or three and why persistent deficits of electron-type neutrinos exist in all solar-neutrino experiments. In line with the theory "neutrino temporal oscillation", the fundamental error is to believe that the neutrino and the photon follow the same transverse path, and that it is the shortest way. The longitudinal path (straight line) is shorter than the traverse path (the curve). The solar neutrino would have a longitudinal wave and its time would be about one third of the time of the photon. The second of the neutrino is therefore approximately one third of the second of the photon (or the Newtonian second). If $1 t_{o}$ is the second of the neutrino, then $t$, or $1 \pi t_{o}$, is the second of the photon $\left(1 \pi t_{o}=\right.$ $1 t$ ). During one second of the photon, the neutrino will have travel $1 \pi t_{o} \mathcal{c}$, say $\pi$ times more distance in straight line than the photon $\left(1 t / \pi=1 t_{o} c\right)$. As physics uses the ordinary transverse second of the photon which is $\sim 3.1416$ times longer than the longitudinal second to calculate the neutrino flux, it appears that the neutrino flux for the distance $1 t_{o} c$ will be about 3 times less dense, because the flux of neutrinos expected by the usual second of the light is spread over a radial path two or three times more distant.

In short, physicists have predicted detect in one "ordinary" second a number of electron neutrinos consistent with physical models of the Sun's interior. Only a third to half the predicted number of neutrinos has been detected. The theory of neutrino temporal oscillation, without requiring a neutrino rest mass, explains the difference like this: the neutrino flux travels radially, not transversely, which means a solar neutrino flux anticipated in an ordinary second divided by 
a number between two or three.

Imagine that a flux of 730 million solar neutrinos per second, having theoretically traveled through a transverse wave, is expected on Earth as predicted from the luminosity of the Sun. We can write

730 million $\sin 90^{\circ}=$ flux of 730 million neutrinos per second.

If the flux measured directly is 243 million neutrinos, i.e. one third of what was expected; we are entitled, to explain the difference, to assume that the neutrinos traveled a less transverse, more longitudinal wave, as if the nodes were stretched radially. Depending on the flow detected we can calculate

730 million sin $19.451^{\circ}=$ flux of 243 million neutrinos per second.

\subsection{Supernova 1987A}

In February 1987, SN 1987A was the first nearby supernova that could be seen well since 1604. It was located about 168,000 light years (ly) from Earth in the Large Magellanic Cloud, a small galaxy gravitationally linked to the Milky Way. The energy calculated to be produced from the collapse of type II supernovae is almost 1000 times larger than that observed as light. Standard astrophysical theory indicates that more than 99 per cent of the energy is emitted in the form of neutrinos [22] and holds that a collapsing star should release a burst of neutrinos before the light from the explosions.

The Mont Blanc team believed that they had discovered such a burst. On 24 February 1987, the Italian/Soviet collaboration was the first to report a burst of neutrinos from SN 1987A, detected at their underground observatory at Mont Blanc, after other astronomers had reported optical observation of the supernova [23]. But four and a half hours (h) after the Mont Blanc burst, which consisted of five events over several seconds, a series of pulses in two water Cerenkov detectors were recorded independently, Kamiokande [24] in Japan, IMB [25] in the United States and also by the Baksan detector in Russia. In all, 24 neutrinos were captured. Given both bursts, $7.7 \mathrm{~h}$ had elapsed before the first light was observed [26] [27].

According to the basic theory of stellar collapse, there is an expected time delay of approximately $3 \mathrm{~h}$ between the collapse of the core and the production of visible light at the surface of the star, due to the propagation of a shock wave through the stellar material. How come that the first neutrinos of the supernova 1987 A arrived $7.7 \mathrm{~h}$ before the first photons? The currently-accepted interpretation of this data is that the first burst of neutrinos must not have been associated with the supernova because there is no conventional explanation for how the neutrinos could have arrived at that time. In addition, the fact that the first burst of neutrinos was only detected by the Mont Blanc detector and not the other two detectors, which were assumed at the time to have higher sensitivities, further suggested that the first burst of neutrinos must have been an anomaly that was not associated with Supernova 1987A. This suggests that the first observation of the visible light from the supernova is compatible only with the second burst of 
neutrinos that occurred about three $\mathrm{h}$ before, which corresponds to the time for any light produced inside the star to be prevented, due to the diffusion, from reaching immediately the surface.

Nevertheless, we agree with some experts in the field who consider the origin of the first burst of neutrinos to be an open question because the probability of such an event having occurred at random has been estimated to be less than $10^{-4}$ [28] [29]. The material used in the Mont Blanc detector was different from that used in the other two detectors and the expected sensitivity of detection for the kind of neutrinos in the first burst has been estimated to be a factor of 20 higher in the Mont Blanc detector than the other detectors, which is consistent with the observations [29].

\subsubsection{Scenario of a Double Collapse}

The possibility was expressed that both the Mont Blanc detection and the later bursts recorded simultaneously in the United States and Japan, could have been genuine events linked to SN 1987A [28]. This would require the star to have collapsed initially to a neutron star, releasing low-energy neutrinos picked up at Mont Blanc, but below the energy threshold on the IMB and Kamiokande devices. A second collapse to a black hole would then explain the neutrino burst recorded by IMB and Kamiokande. At Mont Blanc, this burst may have been indistinguishable from the background noise [30] [31].

This scenario is not expected from the models which predict only a single neutrino burst from a collapsing star and which anticipate the first observation of visible light from the supernova approximately $3 \mathrm{~h}$ after the burst of neutrinos. It is the expected time delay between the collapse of the core and the production of light at the surface of the star due to the propagation of a shock wave through the stellar material. The usual interpretation of this data is that the first burst of neutrinos must not have been associated with the supernova because there is no conventional explanation for how the neutrinos could have arrived at that time. Only the observed by IMB and Kamiokande $3 \mathrm{~h}$ fit with the conventional models.

\subsubsection{The Theory of Neutrino Temporal Oscillation Justifies the Scenario of a Double Collapse}

The theory of neutrino temporal oscillation offers an adequate explanation for the possibility of a double collapse of the core and the observations associated with SN 1987A. According to this theory, the neutrino is moving in a longitudinal wave, that is, the oscillatory motion of the neutrino is in the same direction that the wave is traveling. As mentioned earlier, the second of the neutrino belongs to the longitudinal wave, and is about one third $(1 / \pi)$ of the Newtonian second, which is linked to transverse wave. It means that the neutrino browses $\pi$ times more length in a radial path than the photon in a transverse path.

The two bursts of neutrinos from SN1987A were captured in longitudinal time, that is to say in the time associated with the longitudinal wave, while physicists believed to have captured them in transverse waves that are within the 
transverse electromagnetic wave. This means that the $7.7 \mathrm{~h}$ between the first burst at Mont Blanc and the appearance of light are in longitudinal time, not in transverse time. 7.7 longitudinal $\mathrm{h}$ translate into 2.45 transverse $\mathrm{h}\left(7.7 \mathrm{~h} t_{o} / \pi=\right.$ $2.45 \mathrm{~h} t$ ). These $2.45 \mathrm{~h}$ correspond to the time predicted from the standard models, that is to say the approximate $3 \mathrm{~h}$ for the light to occur on the surface. This is the anticipated collapse of the star into neutron star.

It took $4.7 \mathrm{~h}$ between the second burst of neutrinos observed by Mont Blanc and the second burst of neutrinos observed by Kamiokande and IMB. These longitudinal $4.7 \mathrm{~h}$ are translated into transverse $1.5 \mathrm{~h}\left(4.7 \mathrm{~h} t_{o} / \pi=1.5 \mathrm{~h} t\right)$. It means that the second burst of neutrinos, the one of the collapse of neutron star into black hole, starts $1.5 \mathrm{~h}$ after the first collapse. The second burst of neutrinos, $4.7 \mathrm{~h}$ after the first burst, $3 \mathrm{~h}$ before the light, signaled the second collapse of the core. It should be associated to a second production of visible light characterized by the increase in its intensity roughly $4.7 \mathrm{~h}$ after the initial onset of the light.

\subsubsection{Confusion of Running Times}

In our view, the $3 \mathrm{~h}$ between the second burst of neutrinos observed by IMB and Kamiokande and the arrival of light were wrongly coupled with the $3 \mathrm{~h}$ for that the shock wave coming from the core of the supernova reaches the surface. Because the IMB and Kamiokande observations fitted well with theoretical predictions based only on the transversal path of the photon, the general perception among astrophysicists was that the Mont Blanc burst was background noise, most probably caused by penetrating radiation from the surrounding rock, expected about once every three years from random fluctuations [22]. In addition, the detection of two distinct signals implies that the theory predicting only a single neutrino burst from a collapsing star is not right and has suggested that the first burst of neutrinos must have been an anomaly that was not associated with Supernova 1987A [26].

The 2 bursts of neutrinos match the 2 collapses of the supernova. Thus, the arrival time of the first burst of neutrinos is consistent with the observed light curve [26], and the second collapse of the core would have produced an increase in the intensity of the visible light not long after the arrival of first photons. This is consistent with the observation that the light signal increased more rapidly than would have otherwise been expected during that time interval. The theory of neutrino temporal oscillation is hence in reasonable agreement with the experimental observations and it provides a possible explanation for the first burst of neutrinos which is inconsistent with the conventional model of the supernova.

\section{3. “Cosmological” Neutrinos}

Under the Big Bang Standard Model, in the early days of the universe, there were as many particles of matter as there was antimatter. They interacted, met, and annihilated each other to become photons which in turn disappeared to give rise to particle-antiparticle pairs. These photons, later weakened by the expansion of the universe and its cooling, could no longer give birth to particles and antipar- 
ticles. Nature having a preference for matter will ensure that for every billion particles and antiparticles that will annihilate to give 1 billion photons, only one particle of matter will remain, exactly the proportion that is observed in the current universe. All the antimatter disappears [32].

But a species born out of the big bang resisted: cosmological neutrinos and their alter ego, the antineutrinos. Their interactions ceased before their energy, which had become too low, forced them to annihilate like other particles. There are now more than a billion neutrinos and as many antineutrinos for a single proton. The existence and the precise abundance of cosmological neutrinos are confirmed by the study of primordial nucleosynthesis, when a few minutes after the Big Bang the temperature of the universe has dropped below a few billion degrees and protons and neutrons have could combine to form helium nuclei.

One can imagine that when the early universe was hot and dense, neutrinos were moving at the speed of light. In this state, they were not agglomerated under the force of their own gravitational pull. However, after the universe had cooled down and crossed the energy threshold, the neutrinos would have become relativistic, slowed down, and began to move with subluminal speeds. The three known types or "flavors" of neutrinos would therefore have acquired a low mass and the ability to transform from one flavor to another. This phenomenon, the oscillation of neutrinos, was discovered in the late 1990s. As a result, these neutrinos constitute a form of "dark" matter, that is, without significant interaction with matter other than through the force of gravity. Even though at least two of the three types of neutrinos have low mass, and participate in the formation of large structures in the universe, their influence on this formation is negligible. Although their eventual mass is not well known due to their weak interactions with matter, oscillations of neutrinos indicate that the mass of all three types confers on them a contribution of at least $0.13 \%$ in the total energy budget of the universe, where dark matter accounts for about $25 \%$ [33]. Neutrinos from the big bang have an energy that is millions of times lower than that of solar neutrinos and their direct detection is perhaps an unattainable dream.

\subsection{1. "Sterile" Cosmological Neutrinos at the Speed of Light}

There would be, according to us, another "sterile" type of neutrino which would not have slowed down and continued moving at light-speeds after the universe cooled. It would not be able to switch leptonic flavors, like the three types of neutrino with mass (neutrino-electron, neutrino-muon, neutrino-tau), but it would be able to switch bosonic flavors with the photon and the graviton [34]. It would interact less with ordinary matter than the known flavors, which already had become very reluctant to do so after the cooling of the universe.

This would not be the massive neutrino presaged by scientists who think that could explain the mismatch between observations of galaxy clusters and the cosmic microwave background ( $\mathrm{CMB}$ ) if neutrinos were more massive than is usually thought [35]. They suggest the possibility of discovering a right-handed neutrinos impervious to the weak force with a huge mass that does not rely on 
the Higgs field, or to detect a heavy flavor that may emerge from a different mechanism altogether at the extremely high energies of grand unification [2]. "Sterile" cosmological neutrino at the speed of light would be akin to the concept of massless neutrino of the original Standard Model. All along the expansion, always at light speed, the frequency of elusive sterile neutrinos decreases. The lost energy is transformed into mass, clustering along with the rest of the matter, making a larger contribution to the total density of the universe. Besides being a cosmic chameleon which can change bosonic identity, this neutrino would have the peculiarity to follow a longitudinal wave. Thus, if the age of the universe was around 5 billion transverse ly, that would be tantamount to more than 15 billion longitudinal ly ( 5 billion ly $t \times \pi)$. As known, in various ways, but based on electromagnetic waves, astrophysicists have established the age of the universe around fifteen billion ly. This would mean that there are neutrino waves that traveled radially over 45 billion ly, and that the linear radius of the universe would measure more than 45 billion ly $\left(15 \times 10^{9}\right.$ ly $\left.t \times \pi=\sim 47 \times 10^{9} \mathrm{ly} t_{o}\right)$.

We noticed that this last length had a link with an intriguing feature in the WMAP cosmic maps [36]: the early universe does not have a voice on the long wavelengths and does not sound like it would do if the space was apparently Euclidean and infinite. To explain, let's say that CMB temperature fluctuations can be decomposed into a combination of spherical harmonics. The relative magnitude of each spherical harmonic sets the "power spectrum" containing a signature of the geometry of the universe and the conditions at the time of emission of radiation. The power spectrum exhibits a series of peaks when the distance is measured between the regions of the sky of small and medium dimensions. In harmonic analysis of WMAP, these peaks are consistent with what is provided by the "Standard Model" for small angles. For separate regions of more than $60^{\circ}$, there is a loss of power that is not consistent with the predictions of the Standard Model. WMAP observed a quadrupole (harmonic which corresponds to an observation angle of $90^{\circ}$ ) seven times lower than what is expected with $0.2 \%$ probability that this difference occurs by chance. The low value of the quadrupole means it lacks the very long wavelengths [37].

Some cosmologists have proposed to attribute this anomaly to undiscovered physical laws that have governed the early universe. Our explanation for this phenomenon, which seems geometric, hinges on a space model in which large angular scales contain the largest "voids" of which the size of the space imposes a maximum length at the longitudinal wavelength, whence the $\sim 45$ billion ly $t_{o}$.

\subsubsection{New "Bosonic" Flavors: Photonic Neutrino, Gravitonic Neutrino}

We envisage the existence of massless sterile neutrinos, without charge, at the speed of light, under the aspect of a family other than that of the leptons, preferably the bosons, which implies spin quantum numbers with integer values. There are no theoretical arguments which forbid the neutrinos to not have rest mass or to have transitions between various sorts of bosons. Although they remain without rest mass, they have an intrinsic mass (or motion mass) that al- 
lows them to oscillate.

If we consider that the sterile neutrinos propagate at the speed of light, in space or in matter, nothing forbids them to change identities often, to oscillate between two types of bosonic neutrinos: photon and graviton. The oscillation requires the existence of diverse flavors of bosons-neutrinos and differences between the intrinsic masses of the flavors. These alterations are related to the frequency of the oscillations, so that new oscillations measurements in the future could suggest how large the dissimilarities might be. Thus, photons and neutrinos-photons would have photon flavor, gravitons and neutrinos-graviton would have graviton flavor. The change from one flavor to another could provide a coherent explanation for the cosmological waves pattern (electromagnetic waves, gravitational waves, neutrino waves) [34].

\section{Discussion}

We think that the controversial experiment of 2011, carried out over a short distance, would have established the existence of massless sterile neutrinos without charge at the speed of light. During these oscillations neutrinos have disappeared from view. What did they do during this short period of time where they were undetectable?

Some scientists think that photon and neutrino invariably follow the same path, but that the speed of the neutrino is truly "superluminal". Others have raised the possibility that the particle has taken a shortcut through space-time. It's been a few decades that the scientific community is considering the existence of dimensions beyond the three that we perceive. To understand that, imagine that we lived on a sheet of paper two-dimensional, without that our senses reveal the third dimension of space. This sheet is bent and, to go from point A to point $B$, we are obliged to follow its curvature. While if we could take a third dimension, the path from A to B would be shorter. So if the neutrinos are experiencing one (or more) extra dimension to what we perceive, they were able to follow a shorter path than light. Hence the neutrinos apparently faster than light.

According to the theory of "neutrino temporal oscillation", neutrinos at the constant speed of light would follow a shortcut in the space-time of three spatial dimensions that we perceive. It is not the same thing as to take a shortcut in extra dimensions. Imagine that we lived in a tunnel in three dimensions, with our senses conditioned to always use the three dimensions of space. To move forward from point $\mathrm{A}$ to point $\mathrm{B}$, regardless of whether the tunnel is straight or curved, we are obliged to follow the rule of the three dimensions which wants that we move away simultaneously our legs to the left and to the right, bring them back, then make a small jump forward, and continue like this up to point B. While if one could just put one foot before the other in order to take only one dimension, the path would be shortened. Thus, contrary to light (photons), certain particles (neutrinos) would be able to go through one (or two) of the three spatial dimensions we perceive. Neutrinos would have traveled faster than the 
photon, not because they are faster, but because they have taken a shortcut through one dimension among those we perceive. This shorter path of the world in three spatial dimensions is similar to a longitudinal wave.

However, it appears that the most trivial explanation is that of massive neutrinos oscillations. This phenomenon would be deeply related to that of the disappearance of neutrinos: we cannot see the neutrinos during the quantum oscillations, because they move in metamorphosing at a speed under $c$, which remains in the vagueness but gives the certainty of a mass, which does not infringe causality based on the radial arrow of time of special relativity.

In strictly deterministic physics such a ghostly behaviour is as strange as the neutrino oscillations itself. It is legitimate to wonder if the currently accepted interpretation below the speed of light is really final and if behind the apparent rigor of retesting, some experiments do not conceal a part of the real profound nature of the neutrino. It is not only a question to challenge the statistical value of elegant and imprecise formalisms with which theorists of neutrinos juggle, but also to ask whether the interpretation being proposed for the 2011-2012 experience has reached finality and the bottom of things. Several observers have been led through the media to monitor the saga that led to the current interpretation of the neutrino velocity under $c$. They were able to find some weak points, like the optical cable errors which have at first reduced the velocity of propagation of neutrino to that of light, and then that one promptly has put slightly less than the speed of light in a vacuum. It suggests a retest having been oriented by the formalism of the postulates of relativity that, perhaps, paradoxically, does not correspond to the physical reality.

As currently formulated (see Section 3.1), the Standard Model has no explanations for neutrino mass. The original Standard Model prohibited neutrinos from having rest masses. Three types of neutrinos have long been established and, though by quite indirect evidence, they seem to transform into each other. In 1997-98, physicists have theorized that a neutrino must have mass by arguing that the mechanism of transformation does not allow for massless particles. The experiments concerned with oscillations did not make it possible to determine the absolute mass of each of the three types of neutrinos but to measure the difference between their masses.

A particle's total energy or mass-energy includes the particle's rest mass and momentum. Determining what portion of a moving particle's mass-energy comes from its rest mass and what portion is momentum turns out to be a thorny problem with neutrinos. In fact, we should just say that neutrinos oscillate: They change from one flavor to another. And to do this there must be differences between the masses of the different flavors; these differences are related to the frequency of the oscillation, and so the new oscillation measurements begin to suggest how large the differences might be. Neutrino physicists have two ways of observing oscillations: by neutrino disappearance or by neutrino appearance. If they make a beam of neutrinos with a single flavor, then find that some of the 
neutrinos in the beam have disappeared, they can guess that the neutrinos have "oscillated" into a flavor that the detector is not sensitive to. Appearance experiments are more satisfying but much rarer: In this case, they detect a new neutrino flavor that was not produced by the original source. In both cases, the evidence is most convincing if the number of neutrinos varies as a function of distance traveled and energy according to the prediction [4]. The basic strategy for measuring neutrino oscillations seems simple: Take a source of neutrinos, either natural or artificial, let the neutrinos propagate for a known distance, and then measure as much as you can about their energy and flavor. If the amount of a given flavor (as a function of energy and distance) turns out to be what is expected according to the quantum-mechanical prediction that arises from the oscillation hypothesis, we had a spontaneous change of flavor.

In line with the transformist theory (see Section 3.1), two-thirds of the missing solar neutrinos would transform into muon neutrinos and tau neutrinos. Electron detectors can only pick up electron neutrinos, which would explain the deficit of solar neutrinos. The Sudbury Observatory detector in Ontario was designed to detect some of the neutrinos produced by the Sun. It contains heavy water: in a molecule of heavy water the hydrogen atoms $\left({ }_{1} \mathrm{H}^{1}\right)$ of light water are replaced by deuterium atoms $\left({ }_{1} \mathrm{H}^{2}\right)$. In a tank of light water, all neutrinos, regardless of their flavor (electron, muon or tau), can react with an electron and give off a flash of light. But only neutrinos-electrons can react with a deuterium atom, an element in heavy water, and give rise to two protons and an electron. Since the flash of light from this reaction is different from that of light water, physicists are able to determine the proportion of neutrinos-electrons that reach the Earth. Result: they enumerated a third of neutrinos-electrons and two thirds of neutrinos-muons and tau. For the theory of oscillation, this is direct proof of the transformation from one species to another.

Since the reasoning is perfect, experts believe it must be done this way. The fact remains that believing and proving make two. Are we dealing with the expert who overheard the theorist who saw the experimenter who saw with certainty such a neutrino transforming into another type of neutrino? A shortage of neutrinos of some type from the atmosphere and the Sun was recorded compared to what was expected by theory. All the experiments confirmed the phenomenon, not only for the neutrinos detected of natural origin, but also for the neutrinos captured by the detectors which are produced by nuclear power plants in normal operation or those emitted by particle accelerators. To explain the deficit observed in the flow of neutrinos produced by nuclear power plants, the assumption has been made of the presence of a fourth type of neutrino, the "sterile" neutrino, more massive than the three others and which would interact even less with ordinary matter. The major result of these experiments led to the conclusion that the enigma of the neutrino deficit stems from their oscillations and that this transformism can only be explained because they have mass. It is possible that it is wrong and we can see how. 
But before, let's mention that highly qualified people can develop a theory, with relentless rigor, mathematics provided, reasoning without flaw, and yet the whole thing is false because the basic premises, postulated without proven foundation, turn out to be false. It is the same for a technological experiment; we can use advanced technicality, reduce uncertainties to a minimum, go through the stages with consistency, rigor and coordination, obtain an irreproachable result taking into account statistical uncertainties and systematic errors, and yet we can swim in error because of a conceptual error.

The truth is that scientists are unable to measure the energy of a neutrino and to know how far it traveled. Not knowing where he finished in the cycle of oscillations, they cannot calculate the relative proportions of the three flavors. Over large distances and long times, neutrinos oscillate so many time that they cannot keep track of the flavor mix it looks like a blur to them. Instead they take a "statistical average", described by a so-called flavor propagation matrix. From this matrix, astronomers can deduce what an observed ratio must originally have been [10]. Precision is missing over long and short distances, which gives as much certainty for a rest mass as for an intrinsic mass.

The whole system can turn out to be a lie because an alternative has been overlooked. Physicists failed to assume that the fluxes of muon and tau flavors could originate from the cosmos, that they could be cosmic neutrinos having a longitudinal wave as we saw in Section 4.1. According to the theory of temporal oscillation, the neutrino travel time is about a third of the photon travel time. The conceptual error is to believe that neutrino time is the same as photon time: this is why we perceive in a transverse second only one third of the predicted solar neutrinos. The same applies to muon-neutrinos and tau-neutrinos. The deuterium detector captures only one third of the electron neutrinos because it can capture only these. The light water electron detector, capable of capturing all three types of neutrino, captures one-third of what is expected from solar neutrinos, and one-third of what is expected for each of the other two kinds of neutrinos that come not from the Sun but from space. Each type of neutrino contributes one-third of the flux, hence all three-thirds. For the theory of neutrino temporal oscillation, it is the proof of the longitudinal (radial) wave which multiplies by three the ordinary flux.

In short, there is a distinction to be made between the true flow of neutrinos, which we equate to "radialism", and the false flow of neutrinos assimilated to "transformism". Physicists consider that the photon and the neutrino follow the same path. In our opinion, they do not follow the same path: the neutrino follows a radial path while the photon follows a transverse path. The radial path is, linearly (radially) speaking, $\sim$ pi $(\pi)$ times longer than the transverse path; it is like the length of an unfolded circumference becoming a straight line. This radial path of the neutrino is that of the true flux of the neutrinos. With respect to the radial reference, we can say that a photon flux travels one diameter while a neutrino flux travels the length of three diameters. This radial path of the photon, 
used indiscriminately for the neutrino, is that of the false flux of the neutrinos. Since physicists do not differentiate between true neutrino flux and false neutrino flux, and only use the latter, it is no surprise that they almost always get a deficit of about two thirds of solar electron neutrinos.

\section{Conclusions}

We surmise the existence of non-sterile and sterile neutrinos, very light, conform to the Standard Model (original model prohibited neutrinos from having rest masses), but with two novel features: they use a shorter radial path than the photon and have bosonic flavors. We name this phenomenon "neutrino temporal oscillation". It is an alternative to the hypothesis of neutrino oscillation. The latter gives a complicated explanation of the periodic disappearance of neutrinos by allowing the three flavors of neutrinos electronic, muonic and tauic, all supposed of different masses, to metamorphose from one flavor to another. The somewhat ad hoc change in flavor of neutrinos during their journey appears to be supported by theory and therefore has the assent of the majority of cosmologists.

The measures of the observations of neutrinos and antineutrinos, based on the calculation of the probabilities of oscillation, bring into playing several parameters: the differences in mass from one flavor to another (although we always ignore the mass of the flavors, which seems paradoxical), the mixing angles between the different flavors and other complex numbers. They show more visible neutrinos that disappear than invisible ones that appear. These measures are quite indirect evidence of transgender metamorphoses during the journey. They quickly turned into conclusive evidence that can dispense with alternatives. Oddly, however, the observations show that in practice one can explain the detection of neutrinos by assuming that it is, on the one hand, transformist and, on the other hand, governed by longitudinal paths. This would not be so much a statistical error or a systematic error as a conceptual error.

To reconcile theory and observation, the two options seem a priori equally admissible. However, the theory of neutrino temporal oscillation, which implies an intrinsic mass of particles, gives the periodic disappearance of neutrinos a more sensible and fact-compatible explanation. It does not use superluminal speed; neither do the membranes of the eleven-dimensional universe of M-theory, nor the strings of string theory. Its interpretation has the merit of revealing the longitudinal wave which is sorely lacking in current physics while respecting the inviolability of the speed of light of special relativity in our four-dimensional universe. This imbalance, between the "neutrino oscillation" which transforms the flavors of neutrinos and the "neutrino temporal oscillation" which transforms the path of the neutrino over time, can evolve, and possibly even toggle.

\section{Conflicts of Interest}

The author declares no conflicts of interest regarding the publication of this paper. 


\section{References}

[1] Kearns, E., Kajita, T. and Totsuka, Y. (1999) Scientific American, 281, 64-71. https://doi.org/10.1038/scientificamerican0899-64

[2] Hirsch, M., Päs, H. and Porod, W. (2013) Scientific American, 308, 40-47.

[3] Overbye, D. (2011) Tiny Neutrinos May Have Broken Cosmic Speed Limit. IIT-BHU Chronicle, September 22.

[4] Kaneyuki, K. and Scholberg, K. (1999) American Scientist, 87, 222-231. https://doi.org/10.1511/1999.24.817

[5] Glashow, S.L. (1961) Nuclear Physics, 22, 579-588. https://doi.org/10.1016/0029-5582(61)90469-2

[6] Weinberg, S. (1967) Physical Review Letters, 19, 1264-1266. https://doi.org/10.1103/PhysRevLett.19.1264

[7] Salam, A. (1994) Weak and Electromagnetic Interactions. World Scientific Series in 20th Century Physics Selected Papers, World Scientific, Singapore, 244-254. https://doi.org/10.1142/9789812795915 0034

[8] Brumfiel, G. (2012) Neutrinos Not Faster than Light. Nature. https://doi.org/10.1038/nature.2012.10249

[9] Bagdoo, R. (2015) Recycled Relativity. https://vixra.org/abs/1506.0125

[10] Gelmini, G.B., Kusenko, A. and Weiler, T.J. (2010) Scientific American, 302, 38-45. https://doi.org/10.1038/scientificamerican0510-38

[11] Maitte, B. (1981) La lumière, Points. Édition du Seuil, Paris, 159, 233, 236-238.

[12] Whittaker, E.T. (1910) A History of the Theories of Aether and Electricity from the Age of Descartes to the Close of the Nineteenth Century. Dublin University Press Series, Dublin. https://doi.org/10.5962/bhl.title.19630

[13] Radounskaïa, I. (1972) Idées folles. Éditions MIR, Moscou, 15-17, 23, 56.

[14] Trotman, R.E. (1966) Longitudinal Space-Charge Waves. Chapman and Hall Ltd., London.

[15] Bethe, H.A. (1939) Physical Review, 55, 434-456. https://doi.org/10.1103/PhysRev.55.434

[16] Gamow, G. (1938) Physical Review, 53, 595-604. https://doi.org/10.1103/PhysRev.53.595

[17] Trefil, J. (1988) The Darkside of the Universe. Anchor Books, Doubleday, New York, 156-157.

[18] Bahcall, J.N., et al. (1995) Nature, 375, 29-34. https://doi.org/10.1038/375029a0

[19] Bethe, H.A. (1991) The Road from Los Alamos. Touchtone Book, Simon \& Schuster, New York, 246-251.

[20] Bahcall, J.N. (1989) Neutrino Astrophysics. Cambridge University Press, Cambridge.

[21] Close, F. (2010) Neutrino. Oxford University Press, Oxford, 29-35.

[22] Bethe, H.A. and Brown, G. (1985) Scientific American, 252, 60-68. https://doi.org/10.1038/scientificamerican0585-60

[23] Aldhous, P. (1991) Nature, 350, 643. https://doi.org/10.1038/350643a0

[24] Hirata, K.S., et al. (1987) Physical Review Letters, 58, 1490-1493. https://doi.org/10.1103/PhysRevLett.58.25

[25] Bionta, R.M., et al. (1987) Physical Review Letters, 58, 1494-1496. 
[26] Arnett, W.D., Bahcall, J.N., Kirshner, R.P. and Woosley, S.E. (1989) Annual Review of Astronomy and Astrophysics, 27, 629.

https://doi.org/10.1146/annurev.aa.27.090189.003213

[27] Franson, J.D. (2011) Apparent Correction to the Speed of Light in a Gravitational Potential.

[28] Panagia, N. (2008) Chinese Journal of Astronomy and Astrophysics, 8, 155.

[29] Chen, P., Bloom, E., Madejski, G. and Patrosian, V. (2004) Proceedings of the 22nd Texas Symposium on Relativistic Astrophysics.

https://www.osti.gov/biblio/878411-proceedings-texas-symposium-relativistic

[30] Imshennik, V.S. and Ryazhskaya, O.G. (2004) Astronomy Letters, 30, 14. https://doi.org/10.1134/1.1647473

[31] Lychkovskiy, O. (2008) Analysis of the SN 1987A Two-Stage Explosion Hypothesis with Account for the MSW Neutrino Flavour Conversion.

[32] Trinh, X.T. (1988) La mélodie secrète. Fayard, Paris, 153.

[33] Riazuelo, A. (2020) Les neutrinos cosmologiques. Pour la Science, Paris, Hors-Série, No. 106, 72, 73.

[34] Bagdoo, R. (2020) Journal of Modern Physics, 11, 616-647. https://doi.org/10.4236/jmp.2020.115041

[35] Jayawardhana, R. (2013) Neutrino Hunters. HarperCollins Publishers Ltd., New York, 14, 110, 172.

[36] Smoot, G. and Davidson, K. (1994) Wrinkles in Time. Harper Perennial, New York, 276, 277, 281-283.

[37] Luminet, J.-P. (2001) L’Univers chiffonné. Folio essais, 444-446. 